WS9-P04

\title{
A Scalable Parallel Edge 3D Finite-lement Approach to Marine Controlled Source Electromagnetic Using a Multifrontal Sol
}

\author{
N. Zhao (Chengdu University of Technology), X.B. Wang* (Chengdu \\ University of Technology) \& C. Qin (Chengdu University of Technology)
}

\section{SUMMARY}

We present a Scalable Parallel edge 3D Finite-Element Approach to marine controlled source electromagnetic using a multifrontal solver.By using domain decomposition of METIS grid partition technology,we make the most of each MPI processors.In orde to decompose the matrix system and solve right sides for different sources we use a massive parallel multifrontal solver implementation (MUMPS). The result of 3D CSEM matches very well with the 2.5D CSEM. 


\section{Introduction}

The subsurface lateral variations of conductivity can be strong. Therefore, 3D Controlled Source Electromagnetic (CSEM) can preferably help in prioritizing compartments to reduce risk of appraisal well location (Fanavoll, S., et al. 2010). For 3D CSEM Forward modeling with edge finite-element, the use of Krylov subspace methods requires special preconditioning to deflate the large kernel of the curl operator, but the appropriate preconditioner is always hard to found. The CSEM multisource forward problem was solved using an iterative method, and the computation time increased linearly with the number of source (right sides). Recent advances in numerical factorization and parallel computational have resulted in factorization algorithms that can be implemented on Electromagnetic 3D problems. Therefore, we can use direct solvers to solve these problems.

The idea of decomposing the matrix system and solving right sides for different sources is not new (Oldenburg et al, 2008; Nuno Vieira da Silva et al, 2012). These authors use the factorization and distribute the computation over multiple frequencies.Recently load-balancing schemes gives further possibility to improve the efficiency of parallel algorithm.

In this paper we extend load-balancing approach to 3D CSEM forward modelling. We developed a modeling scheme for 3D CSEM modeling with the edge finite element in the frequency domain using a massive parallel multifrontal solver implementation (MUMPS). The electric field was decomposed in primary and secondary components to eliminate the singularity originated by the source term. The primary field was computed by analytical methods and the secondary field was computed discretizing a second-order partial differential equation for the electric field with the edge finite element. The calculation area use domain decomposition of METIS grid partition technology. The solution to the linear system of equations was obtained using a massive parallel multifrontal solver.

\section{Method}

This paper has proposed a method of using the Domain-Decomposition method (Figure 1). In this way the load balanced cluster efficiency can be maintained. Firstly, we partition the domain into several parts (usually equal to the number of MPI processors) using METIS (Figure 2), and then each process assembles its own contribution to the global linear system. The global linear system is solved by iterative methods or direct methods. Here, we use the parallel direct method provided by MUMPS. Finally, we compute the potential fields (electrical, magnetic fields) using the previously solved solutions.
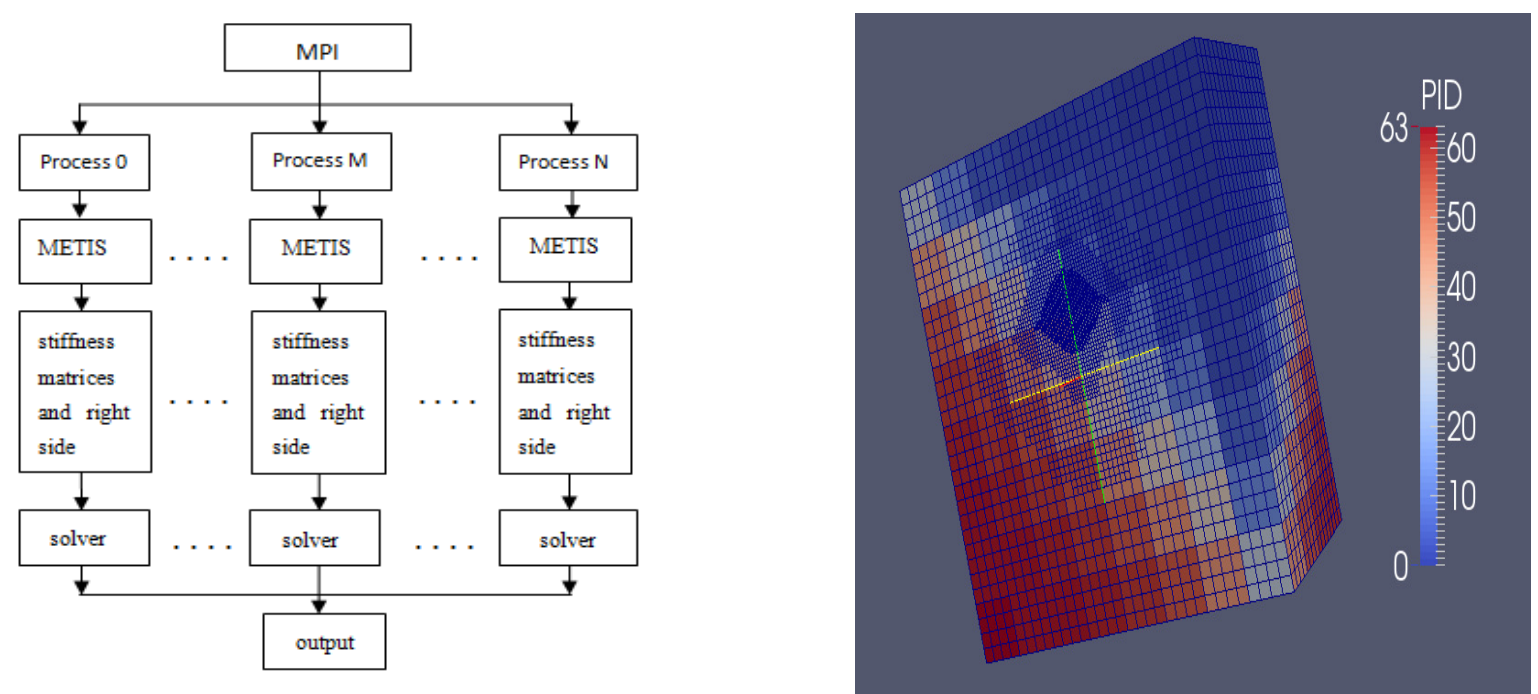

Figure 1 The computing flow chart of our implementations. Figure 2 Meshes partitioned by METIS. 


\section{Examples}

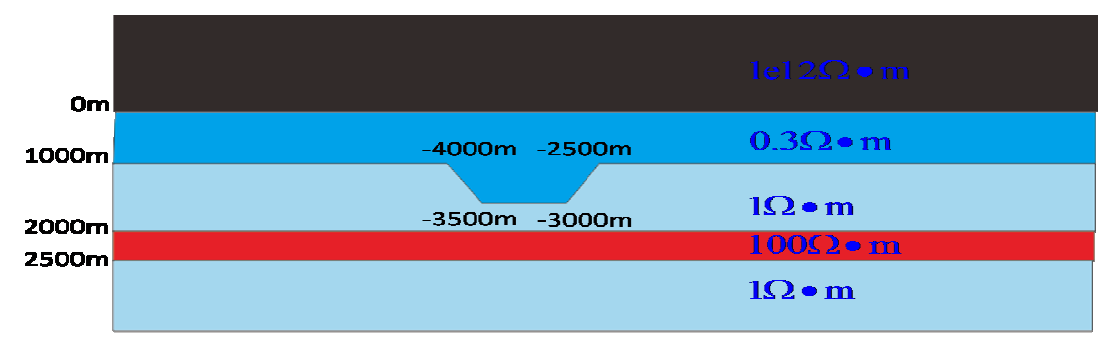

Figure 3 Illustration of the $2 D$ model used to validate the proposed $3 D$ finite element scheme.
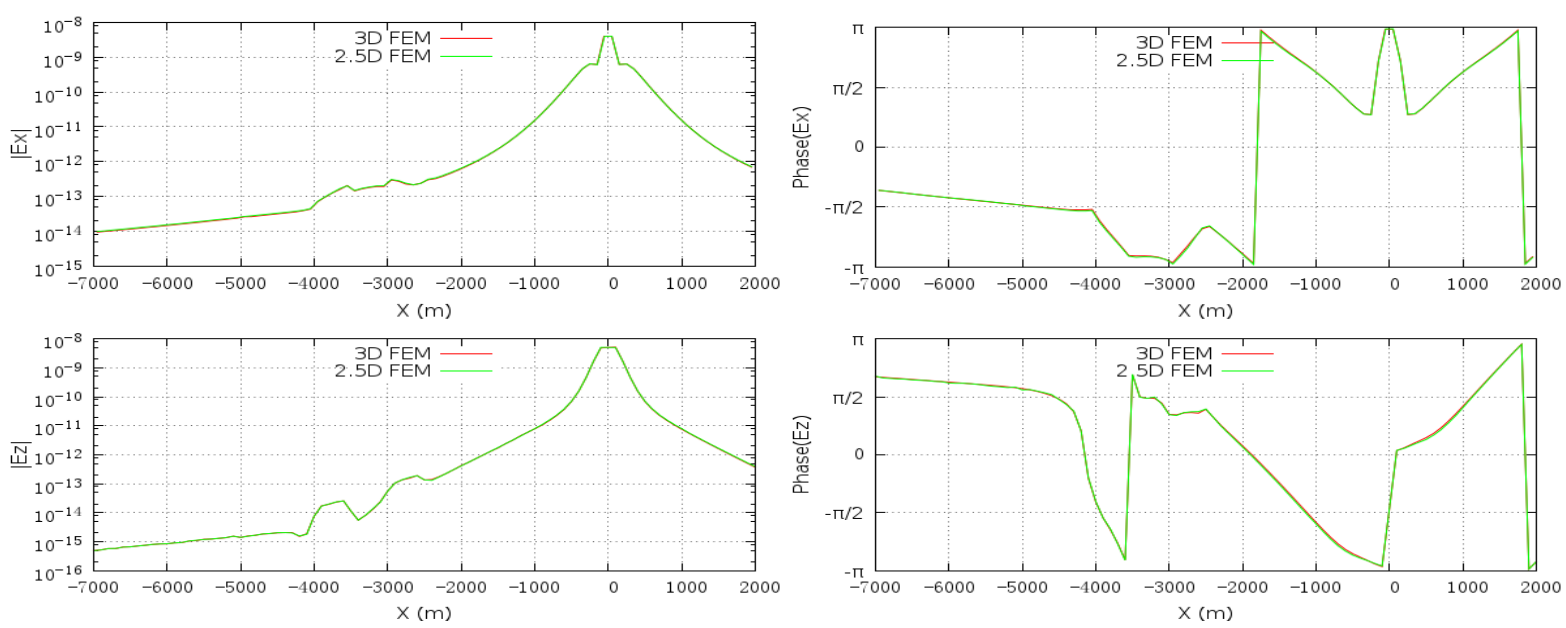

Figure 4 Comparison of the electric field responses for the model in Figure 3 calculated using the $3 D$ vector finite element method (red line) and the 2.5D finite element solution of K. Key (2009)by BGP forward data(green line). The field values are plotted along the receiver line $y=0 \mathrm{~m}$, for both components. This figure shows a very good agreement between our $3 D$ vector finite element solutions and 2.5D finite element solutions.

\section{Conclusions}

We propose 3D CSEM forward modeling by decomposing the electric field in primary and secondary components.The secondary component was computed discretizing the equation for the electric field with the edge finite element.We use METIS with highly efficient load-balancing scheme to raise the parallel efficiency.The linear system of equations is solved using a multifrontal solver.The numerical results in this paper showed that the new parallel algorithm is robust and efficient.

\section{Acknowledgements}

The authors thank BGP and Henan Polytechnic University of high performance computing platform.

\section{References}

Fanavoll, S., et al. [2010] Controlled source electromagnetic technology and hydrocarbon exploration efficiency. First Break, 28(5), 61-69.

Oldenburg, D.W., Haber, E. and Shekhtman, R. [2008] Forward modeling and inversion of multisource TEM data, $78^{\text {th }}$ Annual SEG Meeting, Expanded Abstracts, 559-563.

Vieira da Silva, Nuno, Morgan, Joanna V., MacGregor, Lucy and Warner, Mike [2012] A finite element multifrontal method for 3D CSEM modeling in the frequency domain. Geophysics, 77(2), E101-E115. 